\title{
The Rotational Spectrum of Propane Centrifugal Distortion Analysis
}

\author{
G. Bestmann and H. Dreizler \\ Abteilung Chemische Physik, Institut für Physikalische Chemie der Universität Kiel, \\ West Germany
}

J. M. Vacherand, D. Boucher, B. P. van Eijck, and J. Demaison

Laboratoire de Spectroscopie Hertzienne associé au C.N.R.S., Université de Lille I, France

Z. Naturforsch. 40 a, $508-510$ (1985); received February 18, 1985

\begin{abstract}
The ground state rotational spectrum of propane has been investigated between 6.4 and 26.5 $\mathrm{GHz}$ with a microwave Fourier transform spectrometer and between 140 and $300 \mathrm{GHz}$ with a millimeter-wave spectrometer. High $J$ transitions have been measured and fitted to a centrifugally distorted Hamiltonian including some sextic coefficients. The results of the analysis are sufficiently accurate for the prediction of all strong transitions of astrophysical interest.
\end{abstract}

The ground state rotational spectrum of propane $\left(\mathrm{CH}_{3} \mathrm{CH}_{2} \mathrm{CH}_{3}\right)$ was first measured by Lide [1] who determined the dipole moment, the rotational constants for several isotopic species and a complete $r_{\mathrm{s}}$ structure. The dipole moment was then remeasured with a greater accuracy using a parallel plate cell [2]. The first two torsional excited states were measured by Scharpen [3] and later analyzed by Hirota et al. [4] and by Trinkaus et al. [5]; the barrier to internal rotation is about $13.2 \mathrm{~kJ} / \mathrm{mole}$ (3.2 kcal/mole).

Propane has already been identified in the Voyager IR spectrum of Titan [6] and tentatively in that of Saturn [7]; it presence in interstellar space is also probable. But only seven transitions with $J \leqq 6$ have been measured for the ground state of the most abundant isotopic species, which is insufficient to determine the centrifugal distortion constants and to predict a rotational spectrum.

For these reasons we have measured the rotational spectrum of propane and carried out a complete centrifugal distortion analysis so that accurate measurements and predictions would be available for the radio astronomers.

Detecting high $J$ transitions with a Stark spectrometer would be difficult due to the very small value of the dipole moment $(0.085 \mathrm{D},[2])$.

The measurements were made with a microwave Fourier transform (MWFT) spectrometer [8,9a-c] in the region 6.4 to $26.5 \mathrm{GHz}$ and a video or source

Reprint request to Dr. J. Demaison, U.E.R. de Physique Fondamentale, P5, Université de Lille I, F 59655 Villeneuve d'Ascq, Cedex (France). modulation spectrometer [10] in the region 140 to $300 \mathrm{GHz}$. The measurements with the MWFT spectrometer were made at temperatures of approximately $-40^{\circ} \mathrm{C}$ and pressures below $2 \mathrm{mT}$, with the millimeterwave spectrometer all spectra were measured at $-60^{\circ} \mathrm{C}$ and pressures of about $20 \mathrm{mTorr}$. The accuracy is about 10 to $20 \mathrm{kHz}$ for the MWFT spectrometer and between 30 and $150 \mathrm{kHz}$ for the millimeterwave spectrometer.

The measured lines are given in Table 1. Almost all the centimeterwave lines measured by MWFT were found split by internal rotation. On the other hand no splitting could be satisfactorily resolved in the millimeter region due to Doppler broadening. The internal rotation analysis will be reported elsewhere [11]. For the split lines the unperturbed frequency $v_{0}=\left(v_{\mathrm{AA}}+2 v_{\mathrm{AE}}\right) / 3$ has been used. To derive the molecular parameters the spectrum was fitted to the Hamiltonian of Watson (Eq. (68) of [12]) using the $I^{\mathrm{r}}$ representation in the $A$-reduction (Program ZFAP6, Author V. Typke). The rotational constants, the quartic and four sextic centrifugal distortion constants were determined. They are given in Table 2 together with their standard deviation. A list of predicted lines may be obtained upon request to one of the authors (J.D.).

\section{Acknowledgements}

We thank Dipl. Chem. W. Stahl and Dr. W. Lalowski for measurements at the beginning of the work. This investigation has been supported in part by the Deutsche Forschungsgemeinschaft, the Fonds der Chemie, the Centre National de la Recherche Scientifique (A.T.P. molécules interstellaires) and the Region Nord/Pas-de-Calais. 
Table 1. Measured ground state transitions of propane (in $\mathrm{MHz}$ ).

\begin{tabular}{|c|c|c|c|c|c|c|c|c|c|c|c|c|c|c|c|}
\hline$J^{\prime}$ & $K_{\mathrm{a}}^{\prime}$ & $K_{\mathrm{c}}^{\prime}$ & $J^{\prime \prime}$ & $K_{\mathrm{a}}^{\prime \prime}$ & $K_{\mathrm{c}}^{\prime \prime}$ & $f_{\text {obs }}$ & $f_{\text {calc-obs }}$ & $J^{\prime}$ & $K_{\mathrm{a}}^{\prime}$ & $K_{\mathrm{c}}^{\prime}$ & $J^{\prime}$ & $K_{\mathrm{a}}^{\prime \prime}$ & $K_{\mathrm{c}}^{\prime \prime}$ & $f_{\text {obs }}$ & $f_{\text {calc-obs }}$ \\
\hline 1 & 1 & 0 & 1 & 0 & 1 & 21748.336 & 0.026 & 16 & 5 & 11 & 15 & 6 & 10 & 22862.335 & -0.014 \\
\hline 2 & 0 & 2 & 1 & 1 & 1 & 11013.957 & -0.022 & 16 & 5 & 12 & 15 & 6 & 9 & 22786.504 & 0.008 \\
\hline 2 & 1 & 1 & 2 & 0 & 2 & 22769.712 & 0.021 & 17 & 2 & 15 & 16 & 3 & 14 & 211766.984 & 0.065 \\
\hline 3 & 1 & 2 & 3 & 0 & 3 & 24365.424 & 0.025 & 17 & 2 & 16 & 16 & 1 & 15 & 280971.706 & 0.047 \\
\hline 5 & 1 & 4 & 4 & 2 & 3 & 22933.883 & -0.047 & 17 & 3 & 14 & 16 & 4 & 13 & 142672.637 & 0.045 \\
\hline 5 & 3 & 2 & 6 & 2 & 5 & 11319.926 & 0.089 & 17 & 2 & 16 & 17 & 1 & 17 & 140620.619 & 0.034 \\
\hline 5 & 3 & 3 & 6 & 2 & 4 & 8954.356 & 0.056 & 18 & 2 & 17 & 17 & 1 & 16 & 293185.436 & 0.048 \\
\hline 6 & 5 & 2 & 5 & 4 & 1 & 286689.990 & -0.048 & 18 & 6 & 13 & 17 & 7 & 10 & 12061.849 & 0.041 \\
\hline 7 & 2 & 5 & 6 & 3 & 4 & 8308.367 & -0.075 & 18 & 6 & 12 & 17 & 7 & 11 & 12070.750 & 0.032 \\
\hline 7 & 3 & 4 & 6 & 2 & 5 & 218618.306 & -0.035 & 18 & 1 & 17 & 18 & 0 & 18 & 140017.440 & -0.094 \\
\hline 7 & 3 & 5 & 6 & 2 & 4 & 216117.906 & -0.032 & 18 & 8 & 10 & 19 & 7 & 13 & 14825.050 & -0.008 \\
\hline 7 & 4 & 4 & 8 & 3 & 5 & 20658.616 & 0.084 & 18 & 8 & 11 & 19 & 7 & 12 & 14824.591 & -0.015 \\
\hline 7 & 4 & 3 & 8 & 3 & 6 & 21001.378 & 0.058 & 19 & 0 & 19 & 18 & 1 & 18 & 287574.584 & 0.065 \\
\hline 8 & 2 & 6 & 7 & 1 & 7 & 211481.852 & 0.047 & 19 & 1 & 18 & 18 & 2 & 17 & 289146.451 & 0.072 \\
\hline 8 & 2 & 6 & 7 & 3 & 5 & 26133.102 & -0.046 & 19 & 1 & 19 & 18 & 0 & 18 & 288365.749 & 0.048 \\
\hline 8 & 2 & 7 & 7 & 3 & 4 & 19250.841 & -0.063 & 19 & 3 & 17 & 18 & 4 & 14 & 145856.220 & -0.011 \\
\hline 8 & 4 & 4 & 8 & 3 & 5 & 148136.610 & -0.146 & 19 & 3 & 17 & 19 & 2 & 18 & 143622.180 & -0.014 \\
\hline 9 & 1 & 9 & 8 & 0 & 8 & 146601.280 & -0.019 & 21 & 4 & 18 & 20 & 5 & 15 & 147044.370 & 0.089 \\
\hline 9 & 4 & 5 & 8 & 3 & 6 & 291944.025 & 0.013 & 21 & 9 & 13 & 22 & 8 & 14 & 9282.039 & 0.023 \\
\hline 9 & 4 & 6 & 8 & 3 & 5 & 291586.293 & 0.000 & 26 & 9 & 18 & 25 & 10 & 15 & 12435.726 & 0.026 \\
\hline 9 & 4 & 5 & 9 & 3 & 6 & 147728.480 & -0.075 & 26 & 9 & 17 & 25 & 10 & 16 & 12435.726 & 0.067 \\
\hline 10 & 0 & 10 & 9 & 1 & 9 & 147538.060 & -0.056 & 26 & 1 & 25 & 26 & 0 & 26 & 219527.413 & -0.034 \\
\hline 10 & 3 & 7 & 9 & 4 & 6 & 12381.975 & -0.053 & 26 & 2 & 25 & 26 & 1 & 26 & 220436.493 & -0.006 \\
\hline 10 & 4 & 6 & 10 & 3 & 7 & 147116.440 & -0.086 & 26 & 6 & 20 & 26 & 5 & 21 & 220215.357 & -0.046 \\
\hline 10 & 5 & 5 & 11 & 4 & 8 & 15332.518 & 0.053 & 26 & 11 & 15 & 27 & 10 & 18 & 14384.156 & 0.020 \\
\hline 11 & 2 & 10 & 10 & 1 & 9 & 212660.630 & -0.055 & 27 & 4 & 23 & 26 & 5 & 22 & 293477.608 & -0.064 \\
\hline 11 & 3 & 8 & 10 & 2 & 9 & 288181.882 & 0.057 & 27 & 5 & 22 & 26 & 6 & 21 & 216107.921 & -0.086 \\
\hline 11 & 4 & 7 & 11 & 3 & 8 & 146232.500 & -0.073 & 27 & 3 & 24 & 27 & 2 & 25 & 141387.150 & -0.004 \\
\hline 12 & 3 & 10 & 11 & 2 & 9 & 283543.557 & -0.029 & 27 & 6 & 21 & 27 & 5 & 22 & 217127.058 & -0.019 \\
\hline 12 & 4 & 8 & 12 & 3 & 9 & 145001.150 & -0.055 & 28 & 3 & 26 & 28 & 2 & 27 & 212717.237 & 0.037 \\
\hline 12 & 6 & 7 & 13 & 5 & 8 & 25916.482 & 0.046 & 28 & 5 & 23 & 28 & 4 & 24 & 144760.500 & -0.019 \\
\hline 12 & 6 & 6 & 13 & 5 & 9 & 25926.020 & 0.041 & 28 & 6 & 22 & 28 & 5 & 23 & 213455.847 & -0.047 \\
\hline 13 & 3 & 11 & 12 & 2 & 10 & 295099.457 & 0.018 & 29 & 3 & 27 & 29 & 2 & 28 & 221631.655 & -0.038 \\
\hline 13 & 4 & 10 & 12 & 5 & 7 & 17119.532 & -0.032 & 29 & 5 & 24 & 29 & 4 & 25 & 140209.001 & 0.037 \\
\hline 13 & 4 & 9 & 12 & 5 & 8 & 17441.045 & -0.040 & 29 & 12 & 18 & 30 & 11 & 19 & 8877.344 & 0.050 \\
\hline 13 & 4 & 9 & 13 & 3 & 10 & 143346.340 & 0.007 & 31 & 4 & 28 & 31 & 3 & 29 & 214833.358 & 0.041 \\
\hline 13 & 6 & 7 & 14 & 5 & 10 & 9745.337 & 0.009 & 32 & 4 & 28 & 32 & 3 & 29 & 142955.170 & 0.050 \\
\hline 13 & 6 & 8 & 14 & 5 & 9 & 9725.283 & 0.008 & 32 & 5 & 28 & 32 & 4 & 29 & 211188.559 & 0.102 \\
\hline 14 & 0 & 14 & 13 & 1 & 13 & 211446.958 & 0.038 & 33 & 3 & 30 & 33 & 2 & 31 & 214776.052 & 0.100 \\
\hline 14 & 1 & 14 & 13 & 0 & 13 & 215513.772 & 0.045 & 33 & 5 & 29 & 33 & 4 & 30 & 216255.769 & 0.058 \\
\hline 14 & 2 & 12 & 13 & 3 & 11 & 146021.260 & 0.051 & 34 & 5 & 30 & 34 & 4 & 31 & 221857.648 & -0.028 \\
\hline 14 & 4 & 10 & 14 & 3 & 11 & 141203.180 & -0.082 & 36 & 5 & 31 & 36 & 4 & 32 & 141025.107 & 0.018 \\
\hline 15 & 1 & 14 & 14 & 2 & 13 & 217297.294 & 0.037 & 37 & 5 & 32 & 37 & 4 & 33 & 147362.080 & -0.130 \\
\hline 15 & 7 & 8 & 16 & 6 & 11 & 20374.586 & -0.008 & 39 & 8 & 31 & 39 & 7 & 32 & 296256.010 & 0.014 \\
\hline 15 & 7 & 9 & 16 & 6 & 10 & 20372.438 & -0.005 & 41 & 8 & 33 & 41 & 7 & 34 & 288605.341 & -0.032 \\
\hline
\end{tabular}

Table 2. Rotational, centrifugal distortion constants and correlation matrix for propane ${ }^{\mathrm{a}}$.

\begin{tabular}{lcrrrrrrrrrrrrr}
$A / \mathrm{MHz}$ & $29207.48149(313)$ & 1.000 & & & & & & & & & \\
$B / \mathrm{MHz}$ & 8 & 445.96770 & $(71)$ & 0.294 & 1.000 & & & & & & & & \\
$C / \mathrm{MHz}$ & 7459.00196 & $(73)$ & 0.084 & 0.599 & 1.00 & & & & & & & \\
$\Delta_{J} / \mathrm{kHz}$ & 7.19296 & $(86)$ & 0.176 & 0.743 & 0.743 & 1.000 & & & & & & \\
$\Delta_{J K} / \mathrm{kHz}$ & -26.9670 & $(83)$ & 0.399 & 0.418 & 0.155 & 0.422 & 1.000 & & & & & \\
$\Delta_{K} / \mathrm{kHz}$ & 159.845 & $(94)$ & 0.701 & -0.216 & -0.297 & -0.223 & -0.147 & 1.000 & & & & \\
$\delta_{J} / \mathrm{kHz}$ & 1.39693 & $(42)$ & 0.223 & 0.364 & -0.462 & -0.032 & 0.423 & 0.076 & 1.000 & & & \\
$\delta_{K} / \mathrm{kHz}$ & 3.0585 & $(75)$ & 0.026 & 0.081 & -0.098 & 0.083 & 0.457 & -0.101 & 0.410 & 1.000 & & \\
$\Phi_{J K} / \mathrm{Hz}$ & 0.0296 & $(101)$ & 0.087 & 0.275 & 0.123 & 0.350 & 0.575 & -0.213 & 0.275 & 0.268 & 1.000 & \\
$\Phi_{K J} / \mathrm{Hz}$ & -1.423 & $(168)$ & 0.114 & -0.082 & -0.116 & -0.175 & -0.039 & 0.155 & 0.026 & 0.061 & -0.826 & 1.000 \\
$\Phi_{K} / \mathrm{Hz}$ & 5.52 & $(92)$ & 0.484 & -0.126 & -0.176 & -0.096 & -0.086 & 0.751 & 0.070 & -0.076 & 0.341 & -0.496 & 1.000 \\
$\varphi_{J} / \mathrm{Hz}$ & 0.00368 & $(28)$ & 0.200 & 0.305 & -0.395 & -0.004 & 0.516 & 0.039 & 0.954 & 0.606 & 0.328 & 0.042 & 0.042 & 1.000 \\
\hline
\end{tabular}

a The uncertainties shown in parantheses are in units of the last digit and are standard errors. Representation $I^{r}$ in the $A$-reduction has been used. The standard deviation of the fit was $57 \mathrm{kHz}$. 
[1] D. R. Lide, J. Chem. Phys. 33, 1514 (1960).

[2] J. S. Muenter and V. W. Laurie, J. Chem. Phys. 45, 855 (1966).

[3] L. H. Scharpen, Ph.D. Thesis, Stanford University (1965).

[4] E. Hirota, C. Matsumura, and Y. Morino, Bull. Chem. Soc. Japan 40, 1124 (1967).

[5] A. Trinkaus, H. Dreizler, and H. D. Rudolph, Z. Naturforsch. 28a, 750 (1973).

[6] W. C. Maguire, R. A. Hanel, D. E. Jennings, V. G. Kunde, and R. E. Samuelson, Nature (London) 292, 683 (1981).

[7] R. Hanel, B. Conrath, F. M. Flasar, V. Kunde, W. Maguire, J. Pearl, J. Pirraglia, R. Samuelson, L. Herath, M. Allison, D. Cruikshank, D. Gautier, P. Gierasch, L. Horn, R. Koppany, and C. Ponnamperuma, Science 212, 192 (1981).
[8] G. Bestmann, H. Dreizler, H. Mäder, and U. Andressen, Z. Naturforsch. 35 a, 392 (1980).

[9a] G. Bestmann and H. Dreizler, Z. Naturfosch. 37a, 58 (1982).

[9b] W. Stahl, E. Fliege, H. Dreizler, and R. Schwarz, Z. Naturforsch. 39a, 354 (1984).

[9c] To be published, MWFT spectrometer for K-band.

[10] J. Burie, D. Boucher, J. Demaison, and A. Dubrulle, J. Physique 43, 1319 (1982).

[11] G. Bestmann, W. Lalowski, and H. Dreizler, Z. Naturforsch. 40 a, 271 (1985).

[12] J. K. G. Watson, in: Vibrational spectra and structure (J. R. Durig, ed.), Vol. 6, p. 1, Elsevier, Amsterdam 1977. 